\title{
Aprendizado e mudança em políticas públicas: explorando possibilidades no Modelo de Coalizões de Defesa
}

\author{
Janaina Ma 1 \\ Diego Mota Vieira ${ }^{1}$ \\ 1 Universidade de Brasília / Programa de Pós-graduação em Administração, Brasília / DF - Brasil
}

\begin{abstract}
Este artigo tem como objetivo avançar a discussão sobre a influência do conhecimento e do aprendizado na mudança das políticas públicas, tomando como referência o Modelo de Coalizões de Defesa. Para isso, propomos desvincular a compreensão do aprendizado por meio da mudança em duas perspectivas. Primeiro, propomos apreender a relação entre conhecimento e aprendizagem em políticas públicas, por meio do uso do conhecimento, assumindo que diferentes formas de aprendizagem são possíveis, dependendo do contexto de tomada de decisão. Em seguida, a partir das contribuições da teoria da mudança gradual, propomos usar a noção de dinâmica institucional, a fim de captar o poder explicativo do conhecimento e da aprendizagem tanto em situações de estase quanto de mudança. Esperamos contribuir para diminuir o ceticismo presente na literatura sobre a influência do conhecimento e da aprendizagem no processo de políticas públicas.
\end{abstract}

Palavras-chave: aprendizado em políticas públicas; mudança em políticas públicas; dinâmica institucional.

\section{Aprendizaje y cambio en las políticas públicas: explorando posibilidades en el Marco de las Coaliciones Promotoras}

Este artículo tiene como objetivo avanzar en la discusión sobre la influencia del conocimiento y del aprendizaje en el tema de los cambios en políticas públicas, tomando como referencia el Marco de las Coaliciones Promotoras. Para ello, proponemos desvincular la comprensión del aprendizaje a través del cambio en políticas públicas en dos perspectivas. En primer lugar, nos proponemos aprehender la relación entre conocimiento y aprendizaje en las políticas públicas, a través del uso del conocimiento, asumiendo que son posibles distintas formas de aprendizaje, dependiendo del contexto de toma de decisiones. Luego, con base en los aportes de la teoría del cambio gradual, proponemos utilizar la noción de dinámica institucional, con el fin de captar el poder explicativo del conocimiento y el aprendizaje en situaciones tanto de estasis como de cambio. Esperamos contribuir a reducir el escepticismo presente en la literatura sobre la influencia del conocimiento y el aprendizaje en el proceso de políticas públicas.

Palabras clave: aprendizaje en políticas públicas; cambio en políticas públicas; dinámica institucional.

\section{Policy learning and policy change: exploring possibilities on the Advocacy Coalition Framework}

This article aims to advance the discussion about the influence of knowledge and policy learning on policy change, taking the Advocacy Coalition Framework as reference. We propose unlinking the comprehension of learning through change in two perspectives. First, we suggest apprehending the relation between knowledge and policy learning, through the use of knowledge, assuming that different forms of learning are possible, depending on the context of decision-making. Then, relying on the contributions of the theory of gradual institutional change, we suggest using the notion of institutional dynamics, in order to capture the explanatory power of knowledge and policy learning both in stasis and change situations. We aim to contribute to diminish the skepticism presented in the literature about the influence of knowledge and policy learning in the policy process.

Keywords: policy learning; policy change; institutional dynamics. 


\section{INTRODUÇÃO}

Embora diversas teorias e modelos de análise procurem compreender os mecanismos causais que levam à mudança das políticas públicas (Baumgartner, Jones \& Mortensen, 2014; Kingdon, 2014; Mahoney \& Thelen, 2010), por mais de três décadas os autores do Advocacy Coalition Framework $(A C F)$ têm mostrado um especial interesse pelo papel exercido pelo conhecimento e pelo aprendizado nesse processo (Jenkins-Smith, Nohrstedt, Weible \& Ingold, 2018; Sabatier, 1988; Sabatier \& Jenkins-Smith, 1999).

No entanto, apesar das centenas de estudos empíricos utilizando o modelo, os pesquisadores do $A C F$ ainda lidam com os desafios de estudar o aprendizado no contexto de políticas públicas. As dificuldades incluem a necessidade de avanços teóricos e metodológicos, de modo a melhor compreender o processo de aprendizado, como medi-lo e como ele influencia a mudança nas políticas públicas, dentre outras variáveis consideradas no modelo (Jenkins-Smith et al., 2018; Jenkins-Smith, Nohrstedt, Weible \& Sabatier, 2014). Adicionalmente, os pesquisadores apontam a necessidade de melhor compreender quais e como as características pessoais, os contextos das políticas e os arranjos institucionais favorecem ou dificultam o aprendizado (Moyson, 2017; Weible, 2008).

Este ensaio tem como objetivo oferecer contribuições teóricas e metodológicas ao $A C F$, assumindo que a mudança nas políticas públicas não é a única variável para a apreensão do aprendizado e que o conceito de aprendizado em políticas públicas, como normalmente utilizado pelos pesquisadores do $A C F$, precisa ser expandido, a fim de capturar outras possibilidades, que vão além da alteração no sistema de crenças. Para isso, procuramos suporte em teorias que discutem o uso do conhecimento por atores políticos (Weiss, 1979, 1998) e diferentes formas de aprendizado, de acordo com o contexto de tomada de decisão (Dunlop \& Radaelli, 2013, 2018). Além disso, procuramos explicitar a relação entre aprendizado e estase, explorando as vantagens de ter em conta a dinâmica das políticas públicas, com uma visão mais refinada de mudança e estabilidade das políticas (Mahoney \& Thelen, 2010). A intenção não é desenvolver um novo modelo, mas avançar a partir de um modelo consolidado, capitalizando o conjunto de premissas e conceitos já estabelecidos, para fornecer novos insights aos pesquisadores do $A C F$ interessados no aprendizado em políticas públicas.

O restante do texto está estruturado da seguinte forma. Na seção 2, discute-se a noção de aprendizado no $A C F$ e seus impasses, com base nas aplicações empíricas recentes do modelo. A seção 3 apresenta um conceito ampliado de aprendizado e discute a sua relação com o conhecimento, a partir da classificação dos diferentes tipos de aprendizado e da noção dos usos. A seção 4 explora como a noção de dinâmica institucional ajuda a mapear o relacionamento entre aprendizado e mudança indicado pelo ACF. Além disso, é apresentado um modelo conceitual construído a partir do diálogo entre as teorias analisadas que deve servir de suporte a análises empíricas futuras. A última seção resume as contribuições do modelo proposto e indica algumas proposições para teste empírico.

\section{APRENDIZADO E MUDANÇA EM POLÍTICAS PÚBLICAS NO ADVOCACY COALITION FRAMEWORK: IMPASSES E AVANÇOS}

O ACF é um modelo de análise de políticas públicas que busca compreender o processo das políticas, com foco nas mudanças por que passam e no aprendizado orientado às próprias políticas ao longo de sua implementação. O modelo adota a premissa de que o processo das políticas públicas é complexo e, 
por este motivo, aqueles que desejam influenciá-lo precisam se especializar em subsistemas políticos e formar coalizões. O ACF oferece, assim, uma visão alternativa ao modelo de ciclo de políticas, que permite analisar o processo de maneira mais dinâmica, considerando os processos de formação de agenda, formulação, implementação e avaliação de políticas numa perspectiva não linear (JenkinsSmith et al., 2014; Sabatier \& Jenkins-Smith, 1999, 2007).

Na perspectiva do $A C F$, o indivíduo é dotado de uma racionalidade limitada e tende a simplificar a compreensão do mundo que o cerca, por meio de um sistema de crenças. Esse sistema de crenças é erigido numa estrutura hierárquica tripartite, que agrega as crenças conforme o seu grau de resistência a mudanças e inclui: um núcleo duro (valores mais fundamentais); um núcleo da política (aspectos normativos e empíricos referentes à política pública); e um núcleo de aspectos secundárias (instrumentos da política) (Sabatier \& Jenkins-Smith, 1999; Sabatier \& Weible, 2007).

$\mathrm{O} A C F$ estabelece quatro caminhos para a mudança: mudanças significativas externas ou internas ao subsistema, o aprendizado orientado à política e acordos negociados entre coalizões rivais. Os choques externos referem-se a mudanças nas condições socioeconômicas, na opinião pública, na coalizão governante e em outros subsistemas, enquanto os internos incluem desastres ou grandes falhas que ocorrem no próprio subsistema da política. O efeito desses choques é a mudança na distribuição dos recursos políticos críticos, como o suporte financeiro ou o apoio da opinião pública, o que pode levar à substituição da coalizão dominante. O aprendizado orientado à política é conceituado como "alternâncias duradouras de pensamento ou intenções comportamentais que resultam da experiência e que estão preocupadas com a obtenção ou revisão das normas dos sistemas de crenças dos indivíduos ou da coletividade" (Jenkins-Smith, 2014, p. 199). É mais provável nos aspectos secundários da política, levando, assim, a mudanças de menor porte. Os acordos negociados cobrem as situações em que as coalizões rivais procuram um acordo, diante da presença de impasses que exigem alguma solução. Esse caminho inclui a própria noção de aprendizado e pressupõe a existência um arranjo institucional que favoreça a discussão regulada entre as coalizões rumo a um acordo (Sabatier \& Jenkins-Smith, 2007).

A teoria de suporte ao aprendizado no $A C F$ dá ênfase a quatro categorias de fatores explicativos para o aprendizado: os atributos dos fóruns, o nível de conflito entre as coalizões, os atributos do estímulo e os atributos dos atores. Mais especificamente, o $A C F$ assume que o aprendizado orientado à política é mais provável de ocorrer quando: 1) há um fórum com prestígio suficiente para obrigar a participação dos profissionais das diferentes coalizões e cuja regulação seja feita por normas profissionais; 2) o nível de conflito entre as coalizões é intermediário; 3) o problema é mais tratável, porque dispõe de teorias e dados de suporte; e 4) os atores políticos têm crenças moderadas (Jenkins-Smith et al., 2014).

Embora o $A C F$ não estabeleça um conceito para mudança, ela está associada a mudanças nas regras de políticas ou programas governamentais, na alocação de recursos, na decisão de continuar ou encerrar um programa e, consequentemente, mudanças nos resultados e nos impactos das políticas. O ACF estabelece, ainda, níveis de mudança que podem ser menores ou maiores, a depender do quanto se afastam da política precedente. Como as mudanças requerem alterações nos sistemas de crenças, para o $A C F$ é extremamente difícil que elas ocorram voluntariamente, por isso as informações técnicas podem levar ao aprendizado apenas no que se refere às crenças do nível secundário, levando a mudanças menores. Sabatier \& Jenkins-Smith (2007, p. 198) afirmam que "como é impossível que uma mudança maior venha do próprio subsistema, ela deve vir de uma fonte externa".

A Figura 1 apresenta um diagrama esquematizado desse conjunto de possíveis relações entre conhecimento, aprendizado e mudança, conforme estabelecido pelo ACF. 
FIGURA 1 FATORES EXPLICATIVOS DA MUDANÇA NO ACF

CHOQUES EXTERNOS
CHOQUES INTERNOS
ACORDOS
NEGOCIADOS

NEGOCIADOS

CONHECIMENTO
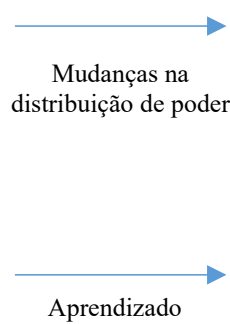

$\begin{array}{ccc}\text { CRENÇAS DO NÚCLEO } & & \text { MUDANÇAS } \\ \text { DURO } & \text { RAIORES } \\ \text { CRENÇAS SOBRE A } & \begin{array}{l}\text { Recursos e } \\ \text { estratégias }\end{array} & \text { POLÍTICA }\end{array}$

CRENÇAS SECUNDÁRIAS

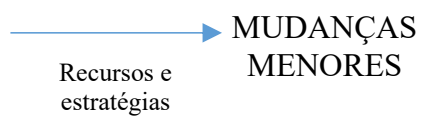

Fonte: Elaborada pelos autores.

A descrição dos caminhos que levam à mudança no $A C F$ revela algumas questões que precisam ser enfrentadas para aprofundar a compreensão sobre a mudança e sua relação com o aprendizado. Uma das críticas feitas ao $A C F$ é que, embora o modelo dê lugar de destaque aos valores e crenças, elas se apresentam muito mais como a cola que une os membros de uma coalizão do que como fatores de mudança (Capano, 2009). O modelo privilegia os choques como principais agentes de grandes mudanças, porque permitem uma reconfiguração na distribuição de poder, ou seja, o poder, mais do que as ideias, explica as grandes mudanças no ACF. Da mesma forma, os acordos negociados após anos de conflito entre as coalizões só ocorrem em resposta a impasses que afetam fatores externos, como a opinião pública, o que gera pressões para a mudança. Em ambos os casos, as mudanças são resultado do enfraquecimento da coalizão dominante ou da substituição da coalização governante. De fato, o ACF assume que as crenças do núcleo duro e da política são muito resistentes a mudança, portanto, é a redistribuição dos recursos que permite que um sistema diferente de crenças tenha mais poder para influenciar as políticas em direção às mudanças desejadas.

Dessa forma, a alteração nas crenças dos indivíduos ocorre apenas como resultado do aprendizado. Os membros das coalizões aprendem quando mudam suas ideias sobre os instrumentos da política, por exemplo, mas como esse processo envolve principalmente os aspectos secundários, ele leva a mudanças de menor porte. Outra questão é que, ao assumir que o aprendizado ocorre quando o conhecimento altera as crenças secundárias, o $A C F$ delimita a noção de aprendizado, sugerindo que quando o conhecimento reforça crenças pré-existentes não há aprendizado. As aplicações empíricas do $A C F$, no entanto, nem sempre dão suporte a esse entendimento. Um estudo conduzido por Leach, Weible, Vince, Siddiki, \& Calanni (2014), em dez parcerias referentes à política de aquicultura marinha nos Estados Unidos, por exemplo, indica que a relação entre aquisição de conhecimento e alteração nas crenças sobre as políticas não é automática. Os autores sugerem que, embora haja uma relação entre as variáveis, a aquisição de conhecimento foi mais comum entre os participantes da pesquisa do que a alteração em suas crenças sobre a política.

Além disso, mesmo quando há uma alteração no sistema de crenças, ela não necessariamente leva a uma mudança na política. Duas razões podem contribuir para explicar esse fenômeno: a inconsistência no processo de aprendizado e o fato de que tanto as mudanças na distribuição de poder, quanto as 
mudanças nas crenças, resultantes do processo de aprendizado, são condições necessárias, mas não suficientes para promover a mudança. A mudança depende, ainda, da mobilização de recursos e de estratégias para o alcance dos objetivos desejados.

A inconsistência do processo de aprendizado é discutida por Moyson (2017). Ele argumenta que, mesmo quando os atores revisam suas crenças sobre a política, eles nem sempre revisam suas preferências políticas de maneira consistente com as novas crenças. Por exemplo, um indivíduo pode acreditar que uma nova evidência prova a ineficiência de uma política do ponto de vista ambiental (mudança na crença sobre a política), mas ainda assim acreditar que a política deve ser mantida, porque os ganhos econômicos superam os prejuízos ambientais (manutenção da preferência política) (Moyson, 2017).

Estudos também mostram que não importa o quão aceita e consolidada esteja uma informação científica, isso pode não ser suficiente para promover as mudanças desejadas, quando as coalizões não são capazes de se organizar adequadamente para influenciar o debate político (Barnes et al., 2016; Khayatzadeh-Mahani, Breton, Ruckert, \& Labonte, 2017; Smith et al., 2015; Ulmanen, Swartling, \& Wallgren, 2015). Khayatzadeh-Mahani et al. (2017), evidenciam que, apesar da quantidade de informação científica sobre os problemas causados pelo tabaco, coalizões pró-tabaco foram capazes de evitar a proibição do fumo de shisha em lugares públicos no Irã. Os autores concluem que o grupo pró- banição não foi capaz de explorar oportunidades, como maior apoio público, para a proibição do uso da shisha. Eles argumentam, no entanto, que houve uma falta de aprendizado devido a uma falta de acordo sobre as evidências. Neste ponto, cabe ponderar se, caso fosse considerada a noção de Moyson de inconsistência entre as crenças e as preferências sobre a política, as conclusões não seriam diferentes. E se a coalizão pró-tabaco aprendeu a partir das evidências, mas não mudou suas preferências por conta das consequências socioeconômicas da proibição? E, ainda, quanto aprendizado teve a coalização pró-banição, apesar de não ter alcançado as mudanças desejadas?

Por outro lado, mesmo quando os parâmetros relativamente estáveis favorecem a estase, a mobilização adequada de recursos e estratégias pode levar a mudanças. Mosley e Gibson (2017) mostram que diferentes tipos de informação, quando combinadas com as narrativas corretas, foram capazes de promover mudanças na política da Califórnia para estender o tempo de permanência dos jovens em lares adotivos. Os estudos tendem a mostrar que a informação é um recurso crucial, mas dependente da capacidade das coalizões de agir estrategicamente para influenciar o processo das políticas.

Conforme observado, os estudos que utilizam o ACF como modelo de análise adotam diferentes desenhos da pesquisa e variáveis para apreensão do processo de aprendizado, chegando a resultados e interpretações diversos. A partir disso, algumas perguntas são relevantes: o aprendizado só ocorre quando há alteração nas crenças sobre a política? Quando o conhecimento serve para reforçar crenças pré-existentes, não há aprendizado? O aprendizado só ocorre quando há mudanças na política? Quando as políticas se mantêm inalteradas, não há aprendizado? Essas perguntas ofereceram os insights para a possibilidade de distinguir dois conjuntos de relações: primeiro, a relação entre conhecimento e aprendizado e, em seguida, a relação entre aprendizado e mudança. Essas possibilidades serão exploradas nas seções seguintes. 


\section{CONHECIMENTO E APRENDIZADO: REVISANDO OS USOS DO CONHECIMENTO}

Os atores políticos estão em contato constante com diferentes tipos de dado, informação e conhecimento, mas certamente nem tudo pode ser levado em consideração durante o processo de tomada de decisão. Não é trivial saber se algo foi realmente aprendido, e é provavelmente por isso que os teóricos de políticas públicas tendem a utilizar a mudança na política como a principal evidência de aprendizado. No entanto, embora a mudança seja uma boa evidência, por questões lógicas, isso não implica que nenhuma lição é aprendida quando nada muda. Assim, na ausência de mudança, o que poderia ser considerado uma evidência de aprendizado? Nesta seção, vamos explorar as possibilidades de considerar o uso do conhecimento pelos atores políticos como evidência de aprendizado.

\subsection{Ampliando o conceito de aprendizado}

O conceito de aprendizado proposto pelo $A C F$ indica a alteração duradoura de pensamento ou comportamento, impactando o sistema de crenças. Hall (1993) aponta que o aprendizado, como convencionalmente entendido, "ocorre quando os indivíduos assimilam uma informação nova, incluindo aquela baseada em experiência, e a aplicam em suas ações subsequentes. [...] O aprendizado é observado quando a política muda como resultado desse processo" (Hall, 1993, p. 278, tradução livre). Apesar de considerar a assimilação de informação, tal conceito atrela o significado de aprendizado à mudança na política.

Dunlop e Radaelli (2013), num esforço de sistematizar a literatura sobre aprendizado em políticas públicas, conceituam o aprendizado como uma "atualização de crenças" (Dunlop \& Radaelli, 2013, p. 600, tradução livre). O termo atualizar, embora inclua a mudança, é mais amplo, pois contempla outros significados como corrigir, revisar, melhorar ou renovar, com base numa informação mais recente sobre determinado assunto. Nessa concepção, entendemos que o aprendizado pode incluir a assimilação de informação que muda ou reforça crenças pré-existentes, o que permite a sua ampliação. Isso pode ser também pensado em termos do que é aprendido ou, simplesmente, das lições do aprendizado. Nas palavras de Rose (1991), "lições constituem o que é aprendido [...] e não requerem uma mudança de comportamento como condição de aprendizado (Rose, 1991, p. 7, tradução livre).

Com base nessa discussão prévia, propomos, então, que o aprendizado consiste na assimilação de conhecimento que promove uma atualização nas crenças do indivíduo. Levamos em consideração a noção geral de conhecimento utilizada pelo $A C F$, que inclui informação técnica, estudos científicos ou experiência passada. Em nossa análise, no entanto, por questões metodológicas, vamos considerar apenas o conhecimento organizado de maneira integrada e coerente, baseado em premissas científicas, como um relatório técnico, um artigo científico, um livro ou um artigo de jornal, desde que possa ser utilizado por um ator político. Entendemos que, especialmente no que se refere à experiência, o conhecimento não sistematizado também é parte importante do processo de aprendizado, como quando os legisladores conversam diretamente com seus eleitores, mas tais formas de conhecimento não serão consideradas na discussão proposta.

\subsection{Evidenciando 0 aprendizado por meio dos usos do conhecimento}

Baseando-se nos trabalhos de Weiss $(1986,1988)$, o ACF assume que, para entender o papel da informação nos processos de mudança, é necessário considerar um período de dez anos ou mais, de modo a capturar sua função iluminadora (enlightenment function) (Sabatier \& Jenkins-Smith, 1999). As noções de uso do conhecimento de Weiss serão consideradas aqui. 
As inúmeras experiências de avaliação de políticas públicas, nas décadas de 1960 e 1970, evidenciaram que os resultados da avaliação não exerciam a esperada influência nas decisões sobre os programas governamentais, como a realocação orçamentária ou a seleção de programas para expansão ou redução (Shadish, Cook, \& Leviton, 1991). Nesse contexto, Weiss passou a reconsiderar como os resultados da avaliação deveriam ser usados na política pública e, assim, a questão do uso tornou-se central ao seu trabalho. Para isso, ela expandiu a compreensão da noção de "uso" da pesquisa social no processo político, oferecendo outros significados que vão além das perspectivas mais lineares de influência da ciência no processo decisório, como provedora de uma verdade absoluta (uso orientado pelo conhecimento) ou de soluções para problemas existentes (uso para solução de problemas) (Weiss, 1979).

Weiss compreende que o conhecimento produzido pela pesquisa pode ser também utilizado politicamente, para embasar posições pré-determinadas (uso político) ou de maneira tática, quando a pesquisa é contratada por burocratas, para passar a ideia de responsabilidade ou de que o problema já está sendo estudado e, assim, responder a pressões e retardar a ação (uso tático) (Weiss, 1979). Certamente, a forma de uso que marca o trabalho da autora, no entanto, é a chamada de enlightenment, que ocorre quando os conceitos e perspectivas teóricas desenvolvidas pelas ciências permeiam o processo decisório político de maneira não linear, desconexa e a longo prazo, gerando um conhecimento que influencia a tomada de decisões ao induzir o modo como as pessoas pensam (Shadish et al., 1991; Weiss, 1979). Essa contribuição não ocorre de forma instrumental, pois não precisa ser compatível com os valores e os objetivos dos tomadores de decisão, podendo desafiar verdades adquiridas e superar valores consolidados e padrões de pensamento (Weiss, 1979).

Nesse sentido, embora o $A C F$ assuma que o aprendizado tende a motivar mudanças nos aspectos secundários do sistema de crenças, a noção de uso iluminador do conhecimento, trazida por Weiss, sugere que ideias mais arraigadas, valores e objetivos dos policymakers também podem ser modificados como resultado do processo de aprendizado. Esse tipo de aprendizado, no entanto, é mais difícil de ser medido e rastreado, pois ele ocorre de maneira não linear, permeando o subsistema político por meio de uma espécie de bricolagem, decorrente de um processo disperso de difusão e assimilação do conhecimento.

Baseando-se nos trabalhos de Weiss e Weible (2008)incorpora a questão dos usos do conhecimento científico no estudo das coalizões, propondo uma simplificação em três tipos: 1) político (political use), quando os tomadores de decisão usam o conhecimento para legitimar posições previamente estabelecidas; 2 ) instrumental (instrumental use), quando o conhecimento é utilizado numa perspectiva racional, para resolver um problema a partir dos resultados da pesquisa; e 3) por aprendizagem (learning use), baseado na ideia de enlightenment, quando a acumulação do conhecimento científico gradualmente altera as crenças dos atores políticos. A associação entre o uso por aprendizagem e a noção de enlightenment, no entanto, traz limitações conceituais, pois relaciona a aprendizagem a apenas um dos tipos de uso do conhecimento, o chamado learning use. Dessa forma, um possível aprendizado ocorrido nos outros tipos de uso (político ou instrumental) não é contemplado.

Embora Weiss trabalhe com o conhecimento científico, pois está interessada no uso dos resultados da avaliação externa das políticas, a própria implementação da política também produz conhecimento. Há uma ambiguidade inerente a qualquer política, decorrente das lacunas existentes entre as regras e o que é interpretado ou implementado. Como as regras não cobrem toda a complexidade do mundo real, os atores que implementam as políticas precisam tomar decisões, baseadas em suas premissas implícitas 
e nos recursos disponíveis (Mahoney \& Thelen, 2010). Assim, durante a implementação, eles mapeiam processos, desenvolvem metodologias, protocolos e sistemas de informação, em resumo, desenvolvem rotinas, nas quais se tornam os maiores especialistas (Lupia \& McCubbins, 1994; Rose, 1991). O conhecimento é institucionalizado por meio da capacitação e do treinamento de novas gerações de burocratas, que se tornam importantes fornecedores de informação. Quando os atores da política usam esse tipo de conhecimento, derivado dos processos de implementação, assumiremos aqui que se trata de um uso procedimental do conhecimento. Dessa forma, propomos que essas quatro categorias de uso podem ser consideradas evidências de aprendizado: iluminadora, política, instrumental e procedimental.

\subsection{Considerando diferentes tipos de aprendizado de acordo com o contexto de tomada de decisão}

Dunlop e Radaelli $(2013,2018)$ oferecem suporte teórico para a nossa proposta de ampliação do conceito de aprendizado, bem como para a relação entre conhecimento e aprendizado por meio do uso. Os autores argumentam que o contexto em que a tomada de decisão ocorre possibilita diferentes tipos de aprendizado, considerando duas dimensões de análise: o nível de tratabilidade do problema e a autoridade/legitimidade dos atores-chave como detentores de conhecimento, conforme a Figura 2.

FIGURA 2 APRENDIZADOS EM CONTEXTOS DE TOMADA DE DECISÃO

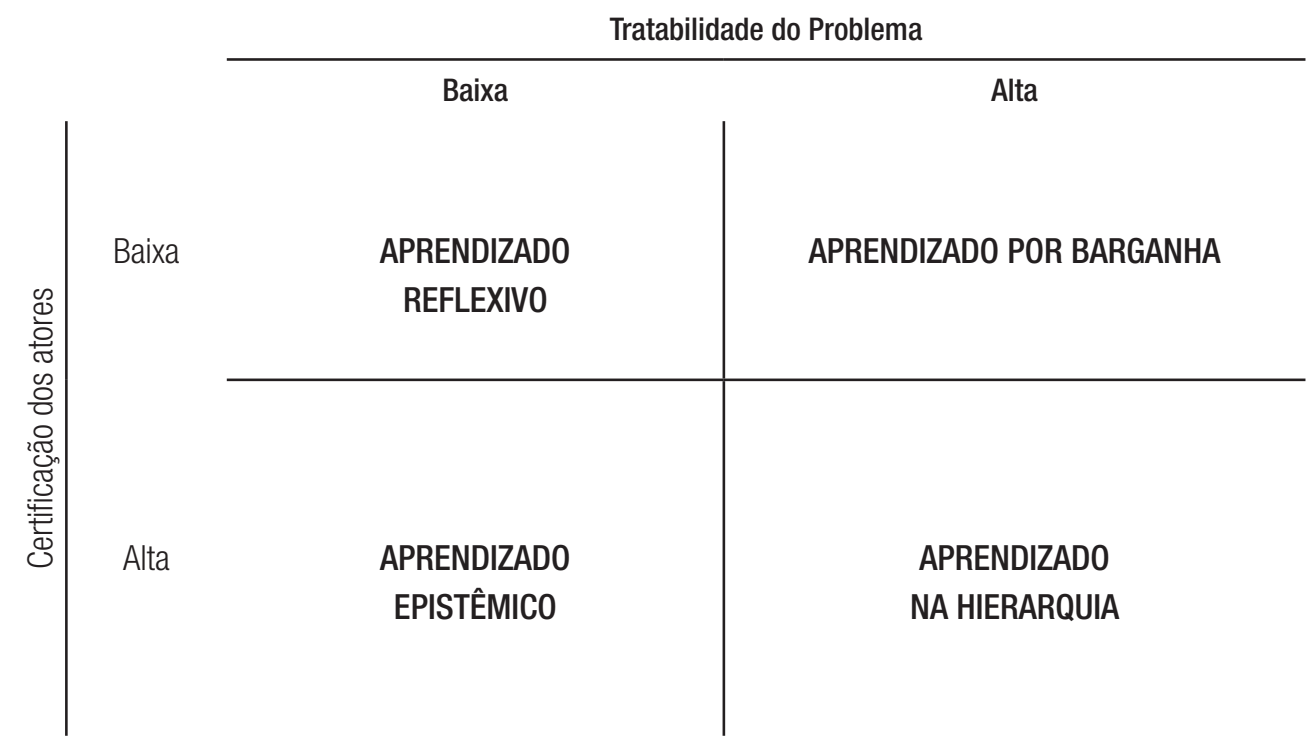

Fonte: Adaptada de Dunlop e Radaelli (2018).

O aprendizado reflexivo ocorre quando o nível de tratabilidade do problema e a certificação dos atores são baixos. Isso significa que o grau de incerteza sobre o problema é alto, ou seja, o conhecimento ainda não está consolidado na comunidade. Nesse contexto, o conhecimento é utilizado com o objetivo de aprofundar a discussão e facilitar a argumentação. $\mathrm{O}$ aprendizado nesse quadrante é geralmente profundo e complexo, pois deve permitir aos atores explorar suas preferências, identidades e estratégias sobre a política (Dunlop \& Radaelli, 2013). 
Para que haja aprendizado, é preciso que haja predisposição dos atores para ouvir o que os outros têm a dizer e reconfigurar suas preferências, além de arranjos institucionais que favoreçam a socialização, a deliberação e a coprodução entre os atores. Nesse caso, o diálogo aberto permite o aprendizado sobre as normas sociais, a reconfiguração da identidade dos atores e da definição do que é apropriado ou não (Dunlop \& Radaelli, 2018).

Nós entendemos que, em contextos de reflexividade, o aprendizado pode ser evidenciado pelo uso iluminador do conhecimento, já que nesse quadrante o conhecimento deve permitir um aprofundamento em questões que ainda não estão tão claras e para as quais os especialistas ainda não têm respostas. Como ele favorece a discussão aprofundada sobre o tema, os atores políticos têm a oportunidade de refletir sobre suas crenças e preferências ao longo do processo político. Isto ajuda a explicar, por exemplo, porque o aprendizado não muda necessariamente os valores a curto prazo, mas exerce uma pressão crucial nestes valores a longo prazo, sem considerar posições e preferências já estabelecidas (Moyson et al., 2017).

O aprendizado epistêmico, por outro lado, ocorre quando o nível de tratabilidade do problema é baixo, mas a certificação dos atores é alta, ou seja, há especialistas com autoridade no tema e legitimidade na comunidade. Nesse caso, o conhecimento é disseminado por um grupo de experts, com o objetivo de estreitar a discussão e alcançar soluções para o problema, que funcionam como uma espécie de professores para os tomadores de decisão (Dunlop \& Radaelli, 2013). Este é o contexto da tomada de decisões baseada em evidências, permitindo o aprendizado sobre a relação entre as evidências e os resultados desejados (Dunlop \& Radaelli, 2018).

O aprendizado epistêmico privilegia o uso instrumental do conhecimento, já que os especialistas no domínio atuam como professores para o tomador de decisões, oferecendo conhecimento pronto para a solução dos problemas existentes. Naturalmente, como pressupõe o $A C F$, os especialistas não são neutros, já que também possuem seus próprios julgamentos sobre a política, e o conhecimento pode ainda ser usado pelos membros das coalizões de maneira enviesada, apenas como munição para o debate. Nós encorajamos os pesquisadores, no entanto, a explorar o uso instrumental do conhecimento como evidência de aprendizado, especialmente entre os membros das coalizões minoritárias.

Fenger e Quaglia (2016), por exemplo, numa análise comparativa do aprendizado ocorrido no setor regulatório da União Europeia, Reino Unido e Holanda, evidenciam que o aprendizado foi limitado porque resultou em mudanças nos instrumentos, mas não nos objetivos da política regulatória. Admitem, no entanto, que muitos stakeholders do setor financeiro haviam evidenciado os riscos de uma regulação branda, mas fatores estruturais possivelmente impediram a tradução desses insights numa regulação mais restritiva. Ao final, os próprios autores sugerem que "desacoplar o aprendizado da mudança em políticas públicas parece um importante passo para se compreender o aprendizado" (Fenger \& Quaglia, 2016, p. 14, tradução livre).

O aprendizado epistêmico também pode ser favorecido em subsistemas nos quais os cientistas são os atores mais interessados numa determinada política e, portanto, os membros mais ativos das coalizões. Souza e Secchi (2014), por exemplo, analisaram o papel da comunidade científica na formulação da política de Ciência e Tecnologia em Santa Catarina, no Brasil, e concluíram que a comunidade científica pôde desempenhar um papel proeminente na formulação da política, ocupando posições políticas e administrativas no governo.

O aprendizado por meio da barganha ocorre quando o nível de tratabilidade do problema é alto, mas a certificação dos atores é baixa. Nesse caso, a administração pública dispõe de um repertório de 
soluções e modos de fazer as coisas, por isso não procura por verdades para solucionar problemas, mas por compreender o ambiente e os limites da negociação. Nesse contexto, aprende-se sobre até que ponto as preferências políticas podem ser negociadas, quais fronteiras não podem ser cruzadas (red lines) e sobre os custos da cooperação, em termos de redistribuição de recursos. Para que isso ocorra, é necessário que as barreiras para a formação de contratos (acordos) e para a agregação de preferências sejam baixas e que haja condições que favoreçam o ajustamento partidário mútuo, de forma transparente para os envolvidos. É importante que a negociação seja permanente, permitindo que ganhadores e perdedores não sejam sempre os mesmos, sob pena de desestimular a interação (Dunlop \& Radaelli, 2013, 2018).

Neste ponto, propomos que o aprendizado por meio de barganha e interação social privilegia o uso político do conhecimento, não no sentido pejorativo, de manipulação do conhecimento para reforçar ou refutar argumentos, mas como forma de compreensão mútua das preferências das coalizões concorrentes, dos limites da negociação e da possibilidade de construção de consensos. O uso político do conhecimento é potencializado nesse quadrante, justamente porque há espaço para visões concorrentes e para a negociação. Vieira (2020), ao analisar o caso da usina hidrelétrica de Belo Monte no Brasil, evidencia como as coalizões favoráveis ao projeto tentaram se unir em torno de uma identidade comum, compartilhando discursos sobre aspectos controversos e alinhando interesses e expectativas.

Cabe argumentar, ainda, que não só as crenças, mas também a dependência interfuncional e a dependência de recursos podem influenciar a forma como as coalizões interagem e cooperam (Weible \& Sabatier, 2005). Elgin (2015), por exemplo, ao examinar o subsistema da política de clima e energia do Colorado, nos Estados Unidos, confirmou que o extremismo nas crenças teve forte efeito positivo na forma como os indivíduos interagiram com aliados ou adversários, mas também concluíram que os indíviduos com níveis mais baixos de recursos organizacionais foram mais propensos a interagir com adversários (Elgin, 2015). Um olhar mais aprofundado dessas interações pode ser uma oportunidade para evidenciar o aprendizado sobre o próprio subsistema político.

Finalmente, o aprendizado na hierarquia ocorre quando o nível de tratabilidade do problema e a certificação dos atores são altos, ou seja, os problemas apresentam um nível baixo de incerteza e risco e há especialistas reconhecidos no tema. Nesse caso, há pouco espaço para a reflexão e para a barganha e o conhecimento se institucionaliza nos processos formais e informais, levando à conformação dos atores (compliance). Nesse contexto, aprende-se sobre o escopo das regras, sua flexibilidade e os custos da não conformidade. Para que ocorra, é necessário que haja uma predisposição dos atores para a conformidade, seja pela lógica do interesse, normativa ou cultural (Dunlop \& Radaelli, 2013, 2018).

Nós sugerimos que o aprendizado em contextos hierárquicos favorece o uso procedimental do conhecimento, relacionado à manutenção das regras, procedimentos e papeis institucionalizados. Esse é o tipo de conhecimento desenvolvido e utilizado por burocratas e técnicos governamentais para manter as complexas rotinas envolvidas na implementação das políticas, que requerem treinamento constante. O conhecimento utilizado como forma de reforçar processos existentes contribui para a institucionalização das regras e pode ser explorado para evitar ou apoiar as mudanças. Problemas de agente-principal podem interferir nesse processo, já que a expertise dos burocratas pode torná-los menos suscetíveis aos contratos desenhados pelos legisladores e a outras características institucionais (Lupia \& McCubbins, 1994). 
O Quadro 1 apresenta um resumo dos tipos de aprendizado e suas principais características.

\section{QUADRO 1 CARACTERÍSTICAS DOS TIPOS DE APRENDIZADO EM DIFERENTES CONTEXTOS DE TOMADA DE DECISÃO}

\begin{tabular}{|c|c|c|c|c|}
\hline $\begin{array}{l}\text { Tipo de } \\
\text { Aprendizado/ } \\
\text { Contexto }\end{array}$ & Na reflexividade & $\begin{array}{l}\text { Em contextos } \\
\text { epistêmicos }\end{array}$ & Na barganha & Na hierarquia \\
\hline $\begin{array}{l}\text { Tratabilidade } \\
\text { do problema }\end{array}$ & Baixa & Baixa & Alta & Alta \\
\hline $\begin{array}{l}\text { Certificação } \\
\text { dos atores }\end{array}$ & Baixa & Alta & Baixa & Alta \\
\hline Metáfora & Diálogo & Sala de aula & Troca & Obediência \\
\hline $\begin{array}{l}0 \text { que é } \\
\text { aprendido }\end{array}$ & $\begin{array}{l}\text { Normas sociais e } \\
\text { identidades; Novos } \\
\text { consensos e novas } \\
\text { definições do que é } \\
\text { apropriado. } \\
\text { Como aprender. }\end{array}$ & $\begin{array}{c}\text { Relações de causa e } \\
\text { efeito; } \\
\text { Conexão entre as } \\
\text { evidências e resultados } \\
\text { desejados. }\end{array}$ & $\begin{array}{c}\text { Preferências políticas } \\
\text { (possibilidades de união } \\
\text { e limites) e custos de } \\
\text { cooperação (alocação de } \\
\text { recursos). }\end{array}$ & $\begin{array}{l}\text { O escopo das regras, } \\
\text { sua flexibilidade e o } \\
\text { que acontece quando } \\
\text { elas não são seguidas. }\end{array}$ \\
\hline $\begin{array}{l}\text { Tomada de } \\
\text { decisão }\end{array}$ & Baseada na comunicação & $\begin{array}{l}\text { Baseada em } \\
\text { conhecimento }\end{array}$ & Baseada na negociação & $\begin{array}{c}\text { Baseada nas } \\
\text { instituições e suas } \\
\text { ambiguidades }\end{array}$ \\
\hline $\begin{array}{l}\text { Gatilhos } \\
\text { institucionais e } \\
\text { pessoais para } \\
0 \text { aprendizado }\end{array}$ & $\begin{array}{l}\text { Arranjos institucionais que } \\
\text { privilegiem a socialização, a } \\
\text { deliberação e a coprodução; } \\
\text { Normas que privilegiem a } \\
\text { competência moral e não } \\
\text { política ou hierárquica; } \\
\text { Predisposição dos atores. }\end{array}$ & $\begin{array}{l}\text { Direitos estatutários de } \\
\text { consulta; } \\
\text { Abordagem pluralística } \\
\text { para o uso da } \\
\text { expertise; } \\
\text { Empreendedores } \\
\text { normativos; } \\
\text { Instituições informais } \\
\text { de colaboração. }\end{array}$ & $\begin{array}{c}\text { Barreiras de contrato e de } \\
\text { agregação de preferências } \\
\text { baixas; } \\
\text { Transparência que favoreça } \\
\text { o ajustamento partidário } \\
\text { mútuo; } \\
\text { Predisposição dos atores } \\
\text { para repactuar assuntos e } \\
\text { trocar de arena. } \\
\text { Permanência da } \\
\text { negociação e alternância } \\
\text { entre ganhadores e } \\
\text { perdedores. }\end{array}$ & $\begin{array}{l}\text { Disposição dos atores } \\
\text { para a conformidade } \\
\text { (compliance), seja } \\
\text { pela lógica do } \\
\text { interesse, normativa } \\
\text { ou cultural. }\end{array}$ \\
\hline $\begin{array}{l}\text { Obstáculos ao } \\
\text { aprendizado }\end{array}$ & $\begin{array}{l}\text { Culturas sem tradição } \\
\text { deliberativa, em que o } \\
\text { acordo é considerado } \\
\text { como perda da honra ou } \\
\text { reputação. }\end{array}$ & $\begin{array}{c}\text { Fragmentação } \\
\text { das comunidades } \\
\text { epistêmicas. }\end{array}$ & $\begin{array}{c}\text { Consolidação de } \\
\text { ganhadores e perdedores; } \\
\text { Baixo custo de defecção, } \\
\text { deixando o ambiente } \\
\text { instável. }\end{array}$ & $\begin{array}{c}\text { Grande quantidade de } \\
\text { veto players. }\end{array}$ \\
\hline $\begin{array}{l}\text { Uso do } \\
\text { conhecimento }^{\mathrm{a}}\end{array}$ & Iluminador & Instrumental & Político & Procedimental \\
\hline
\end{tabular}

aAdaptado de Weiss (1998).

Fonte: Adaptado de Dunlop e Radaelli (2018). 
Em termos de arranjos institucionais, as principais preocupações do ACF permanecem. É importante olhar para as estruturas de oportunidade de longo prazo das coalizões. Os estudos estão longe de serem conclusivos a respeito da influência da abertura do sistema político sobre a facilidade do aprendizado. A falta de arenas para o aprendizado é evidenciada tanto em contextos autoritários (Khayatzadeh-Mahani, Breton, Ruckert, \& Labonte, 2017) quanto em sistemas democráticos (Anderson \& MacLean, 2015; Ulmanen, Swartling, \& Wallgren, 2015). Apenas como exemplo, a experiência de Guangzhou, China, é considerada um marco na gestão e governança urbana, por conta da criação de comitês consultivos, que permitiram solucionar conflitos sociais e aprimorar a comunicação entre os cidadãos e o governo local (Wong, 2016).

Em termos de subsistemas colaborativos ou adversários, pode-se dizer que o aprendizado se beneficiaria de um subsistema colaborativo, mas isso também não é tão claro ainda. Rietig (2018) evidenciou como um subsistema colaborativo se transformou em adversário após o surgimento de novas evidências científicas sobre os impactos ambientais e climáticos negativos dos biocombustíveis de base agrícola.

Finalmente, pode haver alguma relação entre contextos de aprendizagem e a maturidade dos subsistemas, mas ainda é algo a ser explorado. Teoricamente, pelo menos, é razoável considerar que contextos de reflexividade e o uso iluminador do conhecimento só são possíveis em subsistemas maduros. A continuidade das aplicações empíricas que testem as hipóteses sobre o aprendizado do $A C F$, especialmente aquelas relacionadas ao nível de conflito e à existência de fóruns, certamente ajudarão a entender melhor essas questões.

\section{APRENDIZADO E A DINÂMICA DAS POLÍTICAS PÚBLICAS: PROCURANDO 0 ELO PERDIDO}

Após desacoplar a apreensão do aprendizado por meio da mudança, resta compreender como o aprendizado influencia o processo político. Para isso, propomos a observação da dinâmica institucional, considerando os processos de estabilidade e mudança como partes interrelacionadas do mesmo processo de desenvolvimento (Capano, 2009; Real-Dato, 2009). Mais recentemente, o entendimento da mudança como uma ruptura com a estabilidade vem sendo reformulado. Mahoney e Thelen (2010) têm um importante papel nessa discussão, ao assumirem que as mudanças também ocorrem de maneira contínua e gradual. Isso implica que a visão linear de equilíbrio pontuado por momentos de ruptura dá espaço a um processo mais dialético de mudança institucional, em que a ação dos atores em disputa é constante. Dessa forma, tanto a estabilidade quanto a mudança são resultado de tensões em permanente disputa por espaços de poder. Interpretações dominantes das regras precisam ser constantemente legitimadas. Elas não permanecem de maneira pacífica, mas porque se legitimam continuamente frente a interpretações alternativas. Isso expande as possibilidades de compreensão do papel do aprendizado no processo das políticas públicas.

A primeira possibilidade é observar o aprendizado como um dos fatores explicativos da estase. Os estudos do $A C F$ tendem a oferecer explicações para as mudanças, mas não para a estase, geralmente compreendida como resultado da predominância de um certo sistema de crenças. Como a variável dependente é a mudança, quando ela não ocorre, os mesmos fatores explicativos são então considerados ausentes, como a falta de choques, a falta de aprendizado ou a falta de recursos. Conforme discutido, como o aprendizado não implica necessariamente em mudança, há espaço para considerar o aprendizado como um dos fatores explicativos para a estase. Isso ocorre quando os atores políticos usam o que foi aprendido e mobilizam recursos para manter o status quo. 
A segunda possibilidade é considerar o aprendizado como um dos mecanismos que levam a mudanças maiores, algo que não é muito explorado pelos pesquisadores do $A C F$. Uma das críticas feitas ao $A C F$ é que ele dá pouco espaço aos movimentos internos das coalizões para manipular fatores externos ou gerar crises internas que resultam em mudanças maiores (Capano, 2009). A teoria da mudança gradual tem mostrado, no entanto, que os movimentos internos das coalizões são importantes fatores de redistribuição de poder e, consequentemente, de mudança institucional (Mahoney \& Thelen, 2010).

A mudança no sistema de saúde brasileiro de centralizado e restrito para descentralizado e universal é um exemplo desse fenômeno (Falleti, 2010). Embora a maioria das análises prévias tenham oferecido explicações baseadas em conjunturas críticas, nesse caso, a redemocratização, Falleti (2010) demonstra que tal mudança também foi resultado de mudanças graduais, promovidas pelo movimento sanitarista, que se iniciou muito antes da redemocratização, infiltrando o Estado. Esse estudo não explora explicitamente o aprendizado como mecanismo causal da mudança, mas intrinsecamente oferece evidências da importância do aprendizado nesse processo. Como argumenta Falleti, quando a assembleia constituinte discutiu as regras para o novo sistema de saúde,

os sanitaristas já tinham estabelecido tanto as redes quanto a expertise que os deixaram em posição de exercer grande influência, de modo que a codificação e a institucionalização das práticas que eles haviam aperfeiçoado ao longo do período militar foram politicamente possíveis (Falleti, 2010, p. 58, tradução livre).

Essa expertise foi notadamente resultante de longos e intensos processos de aprendizado, derivados da criação de departamentos de medicina preventiva nas universidades brasileiras, da implementação de programas de saúde e saneamento, envolvendo cientistas e servidores públicos, e da existência de fóruns de debate, como as conferências nacionais de saúde.

Assim, baseando-se nas discussões apresentadas, a Figura 3 apresenta uma proposta revisada das possíveis relações entre conhecimento, aprendizado e mudança evidenciadas pelo ACF (Figura 1).

\section{FIGURA 3 FATORES EXPLICATIVOS DA DINÂMICA INSTITUCIONAL}

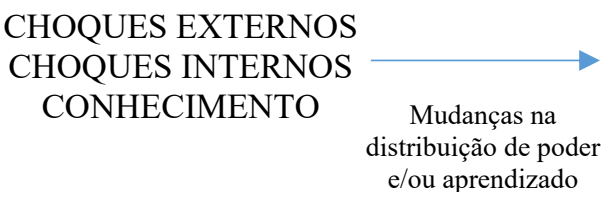

Fonte: Elaborada pelos autores.

\section{ATUALIZAÇÃO NAS CRENÇAS \\ Recursos e estratégias \\ DINÂMICA
INSTITUCIONAL}

Esse diagrama inclui todas as relações do $A C F$, mas abre espaço para outras. Começando pelo fim, a dinâmica institucional inclui mudança e estase como possíveis variáveis a serem explicadas. Considerar a estase como variável dependente requer dar atenção aos movimentos das coalizões para 
manter o status quo. Isso tem sido feito, por exemplo, por pesquisadores que combinam o ACF com a abordagem de análise de narrativas, já que as narrativas são tão importantes para explicar mudanças quanto para explicar a estagnação das políticas (Menahem \& Gilad, 2016; Mosley \& Gibson, 2017). Nós sugerimos que o aprendizado também tem esse poder explicativo, desde que o seu conceito seja ampliado, para incluir qualquer forma de atualização nas crenças.

A atualização nas crenças mantém o significado duplo que a alteração nas crenças possui no $A C F$. Ou seja, ela pode ser o resultado a) da redistribuição de poder, que pode trazer o sistema de crenças de uma coalizão minoritária para uma posição estratégica (os indivíduos não alteram suas crenças), ou b) do processo de aprendizado, quando os membros da coalizão modificam o seu entendimento sobre os problemas e/ou soluções relacionados à política (os indivíduos atualizam suas crenças). A diferença ocorre no último, porque o conceito de aprendizado como atualização de crenças cobre as situações em que os membros de uma coalizão reforçam seus entendimentos sobre a política.

Os choques externos e internos continuam a ser fatores explicativos importantes da mudança. O conhecimento, porém, ganha o poder de promover processos de aprendizado relacionados a qualquer nível do sistema de crenças. A proposta, portanto, não diferencia o poder explicativo dos choques do poder do conhecimento, já que todos os fatores podem levar à atualização das crenças em qualquer nível e influenciar a dinâmica institucional. Essa atualização, como dito antes, pode ser resultado da mudança na distribuição de poder e/ou do aprendizado, que podem se influenciar ou ocorrer simultaneamente, como caminhos diferentes levando ao mesmo resultado (equifinalidade).

Muitas combinações diferentes de caminhos que levam a uma configuração específica da política são possíveis. Em termos de aprendizado, que é o foco deste trabalho, o caminho geral considerado pelo ACF continua contemplado (conhecimento-aprendizado-mudança de menor porte), mas basicamente quatro outras possibilidades são acrescentadas. $\mathrm{O}$ aprendizado hierárquico que pode informar os policymakers; o aprendizado epistêmico que reforça ou revigora crenças existentes; o aprendizado na barganha, que ajuda a construir consensos; e o aprendizado reflexivo, que permite a reconfiguração da identidade dos atores e da sua forma de ver os problemas das políticas e suas soluções. Tais possibilidades só podem ser abertas se considerarmos o aprendizado como atualização das crenças e olharmos para estase e mudança de maneira dinâmica.

Em resumo, esse novo conjunto de relações é uma tentativa de abrir espaço para o aprendizado como um caminho plausível e importante para mudança ou estabilidade, que vai além dos choques externos e internos. O fator explicativo "acordos negociados" não foi explicitado no diagrama, porque entendemos que ele é consequência de outros fatores já presentes no processo. Pode ser resultado de um choque externo, em que uma reconfiguração de poder leva a novos acordos, ou do aprendizado, especialmente em contextos de barganha, quando os membros das coalizões, após aprenderem sobre as respectivas preferências políticas e sobre os custos e limites da cooperação, são capazes de alcançar consenso. 


\section{CONCLUSÃO}

Este ensaio teve como objetivo oferecer algumas contribuições teóricas e metodológicas à discussão sobre a influência do conhecimento e do aprendizado na mudança das políticas públicas, uma lacuna de pesquisa apontada pelos estudiosos do ACF. A proposta apresentada levou em consideração contribuições da literatura sobre aprendizado e mudança em políticas públicas, tomando o $A C F$ como referência de base, sobre a qual os argumentos foram sendo construídos.

Embora a mudança nas políticas seja uma variável tradicional e com poder para evidenciar o aprendizado em políticas públicas, tentamos explorar que outras possibilidades poderiam ajudar a capturar o aprendizado em contextos políticos. Para isso, com base nos trabalhos de Weiss (1998) e Dunlop e Radaelli $(2013,2018)$ propomos apreender a relação entre conhecimento e aprendizado, por meio dos usos do conhecimento, e assumimos a possibilidade de quatro diferentes formas de aprendizado em função do contexto (de reflexividade, epistêmico, por barganha ou hierárquico).

A noção de aprendizado reflexivo ofereceu a possibilidade de compreender o poder do aprendizado para reconfigurar crenças do núcleo duro. A noção de aprendizado epistêmico ajudou a compreender que o aprendizado também pode ocorrer quando os atores políticos reforçam ou revigoram suas próprias crenças. Para isso, foi crucial ampliar o conceito de aprendizado, como normalmente utilizado pelos pesquisadores do $A C F$ (alteração nas crenças), para atualização nas crenças. A noção de aprendizado na barganha ajudou a esclarecer que, durante o processo de negociação, os membros das coalizões também podem aprender e produzir consensos, mesmo que isso não resulte em mudança na política. A noção de aprendizado na hierarquia abriu espaço para o conhecimento produzido durante o processo de implementação, que torna os burocratas importante fonte de informações para os tomadores de decisão. Finalmente, a noção de dinâmica institucional ajudou a evidenciar o poder explicativo do aprendizado tanto em situações de estase quanto de mudança institucional.

Para nortear a aplicação do modelo em estudos futuros, apresentamos a seguir algumas proposições que resumem as considerações apresentadas:

1. Contextos de reflexividade potencializam o aprendizado por meio do uso iluminador do conhecimento, enquanto em contextos de barganha é mais provável que o aprendizado seja observado pela ocorrência do uso político do conhecimento.

2. Contextos epistêmicos potencializam o aprendizado baseado em evidências, revelando um uso instrumental do conhecimento, enquanto em contextos hierárquicos é mais provável que o aprendizado seja observado no uso procedimental do conhecimento.

3. O aprendizado envolve uma atualização nas crenças dos indivíduos e influencia a dinâmica institucional, tanto para promover quanto para evitar mudanças.

Em termos metodológicos, quando propomos considerar os usos do conhecimento em diferentes contextos de tomada de decisão como evidência de aprendizado, nós entendemos que esse tipo de evidência pode ser mais difícil ou acessível, a depender dos dados disponíveis para o subsistema político investigado. É por este motivo que recomendamos focar em conhecimento organizado ou sistematizado, em algum tipo de produto que possa ser utilizado como evidência de conhecimento. Também compreendemos que os quatro contextos de tomada de decisão podem ocorrer simultaneamente no mesmo subsistema, o que pode exigir atenção a diferentes e dispersos tipos de dados e a utilização de diferentes métodos de análise. 
RAP | Aprendizado e mudança em políticas públicas: explorando possibilidades no Modelo de Coalizões de Defesa

Por fim, esperamos que os ajustes teóricos propostos permitam capturar elementos da dinâmica do desenvolvimento das políticas públicas que podem estar sendo desprivilegiados na análise da maneira como as coalizões utilizam o conhecimento, aprendem e mobilizam recursos e estratégias para alcançar seus objetivos. Sem desprezar o poder explicativo dos choques e do caráter distributivo envolvido nos conflitos entre as coalizões, acredita-se que as contribuições aqui apresentadas podem ajudar a diminuir o ceticismo presente na literatura sobre a influência do aprendizado no processo político. 


\section{REFERÊNCIAS}

Anderson, W. F. A., \& MacLean, D. A. (2015). Public Forest Policy Development in New Brunswick, Canada: multiple streams approach, advocacy coalition framework, and the role of science. Ecology and Society, 20(4), 20-32.

Barnes, C., Van Laerhoven, F., \& Driessen, P. P. J. (2016). Advocating for Change? How a Civil Societyled Coalition Influences the Implementation of the Forest Rights Act in India. World Development, 84, 162-175.

Baumgartner, F. R., Jones, B. D., \& Mortensen, P. B. (2014). Punctuated Equilibrium Theory: Explaining Stability and Change in Public Policymaking. In P. A. Sabatier, \& C. M. Weible (Eds.), Theories of the Policy Process (3rd ed., pp. 59-103). Boulder, CO: Westview Press.

Capano, G. (2009). Understanding Policy Change as an Epistemological and Theoretical Problem. Journal of Comparative Policy Analysis: Research and Practice, 11(1), 7-31.

Dunlop, C. A., \& Radaelli, C. M. (2013). Systematising Policy Learning: From Monolith to Dimensions. Political Studies, 61(3), 599-619.

Dunlop, C. A., \& Radaelli, C. M. (2018). The lessons of policy learning: Types, triggers, hindrances and pathologies. Policy and Politics, 46(2), 255-272.

Elgin, D. J. (2015). Cooperative Interactions among Friends and Foes Operating Within Collaborative Governance Arrangements. Public Administration, 93(3), 769-787.

Falleti, T. (2010). Infiltrating the State: The Evolution of Health Care Reforms in Brazil, 1964-1988. In J. Mahoney, \& K. Thelen (Eds.), Explaining Institutional Change: Ambiguity, Agency and Power (pp. 38-62). New York, NY: Cambridge University Press.

Fenger, M., \& Quaglia, L. (2016). The Global Financial Crisis in Comparative Perspective: Have Policy Makers "Learnt Their Lessons"? Journal of Comparative Policy Analysis, 18(5, SI), 502-517.

Hall, P. A. (1993). Policy Paradigms, Social Learning, and the State: The Case of Economic Policymaking in Britain. Comparative Politics, 25(3), 275-296.

Jenkins-Smith, H. C., Nohrstedt, D., Weible, C. M., \& Ingold, K. (2018). The Advocacy Coalition
Framework: An Overview of the Research Program. In C. M. Weible \& P. A. Sabatier (Eds.), Theories of the Policy Process (4th ed., pp. 135-171). New York, NY: Routledge.

Jenkins-Smith, H. C., Nohrstedt, D., Weible, C. M., \& Sabatier, P. A. (2014). The Advocacy Coalition Framework: Foundations Evolutions Ongoing Research. In P. A. Sabatier \& C. M. Weible (Eds.), Theories of the Policy Process (3rd ed., pp. 183-223). Boulder, CO: Westview Press.

Kahan, D. M. (2013). Ideology, motivated reasoning, and cognitive reflection. Judgment and Decision Making, 8(4), 407-424.

Khayatzadeh-Mahani, A., Breton, E., Ruckert, A., \& Labonte, R. (2017). Banning shisha smoking in public places in Iran: an advocacy coalition framework perspective on policy process and change. Health Policy and Planning, 32(6), 835-846.

Kingdon, J. W. (2014). Agendas, alternatives, and public policies (2nd ed.). Harlow, England: Pearson.

Leach, W. D., Weible, C. M., Vince, S. R., Siddiki, S. N., \& Calanni, J. C. (2014). Fostering Learning through Collaboration: Knowledge Acquisition and Belief Change in Marine Aquaculture Partnerships. Journal of Public Administration Research and Theory, 24(3), 591-622.

Lupia, A., \& McCubbins, M. D. (1994). Designing Bureaucratic Accountability. Law and Contemporary Problems, 57(1), 91-126.

Mahoney, J., \& Thelen, K. (2010). A Theory of Gradual Institutional Change. In J. Mahoney \& K. Thelen (Eds.), Explaining Institutional Change: Ambiguity, Agency and Power (pp. 1-37). New York, NY: Cambridge University Press.

Menahem, G., \& Gilad, S. (2016). Policy Stalemate and Policy Change in Israel's Water Sector 19702010: Advocacy Coalitions and Policy Narratives. Review of Policy Research, 33(3), 316-337.

Mosley, J. E., \& Gibson, K. (2017). Strategic use of evidence in state-level policymaking: matching evidence type to legislative stage. Policy Sciences, 50(4), 697-719.

Moyson, S. (2017). Cognition and policy change: the consistency of policy learning in the advocacy coalition framework. Policy and Society, 36(2), 320-344. 
Moyson, S., Scholten, P., \& Weible, C. M. (2017). Policy learning and policy change: Theorizing their relations from different perspectives. Policy and Society, 36(2), 161-177.

Real-Dato, J. (2009). Mechanisms of Policy Change: A Proposal for a Synthetic Explanatory Framework. Journal of Comparative Policy Analysis: Research and Practice, 11(1), 117-143.

Rietig, K. (2018). The Links Among Contested Knowledge, Beliefs, and Learning in European Climate Governance: From Consensus to Conflict in Reforming Biofuels Policy. Policy Studies Journal, 46(1), 137-159.

Rose, R. (1991). What is Lesson-Drawing? Journal of Public Policy, 11(1), 3-30.

Sabatier, P. A. (1988). An advocacy coalition framework of policy change and the role of policyoriented learning therein. Policy Sciences, 21, 129168.

Sabatier, P. A., \& Jenkins-Smith, H. C. (1999). The advocacy coalition framework: An assessment. In P. Sabatier, \& C. M. Weible (Ed.), Theories of the Policy Process (pp. 117-166). Boulder, CO: Westview Press.

Sabatier, P. A., \& Jenkins-Smith, H. C. (2007). The Advocacy Coalition Framework: Innovations and Clarifications. In P. Sabatier (Ed.), Theories of the Policy Process (pp. 189-220). Boulder: Westview Press.

Shadish, W. R., Cook, T. D., \& Leviton, L. C. (1991). Carol H. Weiss: Linking Evaluation to Policy Research. In W. R. Shadish, T. D. Cook, \& L. C. Leviton (Eds.), Foundations of Program Evaluation (pp. 179-224). Newburry Park, London: Sage.

Souza, L. R. de, \& Secchi, L. (2014). A Política Científica e Tecnológica de Santa Catarina: análise a partir do modelo de coalizões de defesa. Revista de Administração Pública, 48(4), 939-960.

Ulmanen, J., Swartling, A. G., \& Wallgren, O. (2015). Climate Adaptation in Swedish Forestry: Exploring the Debate and Policy Process, 1990-2012. Forests, 6(3), 708-733.

Vieira, D. M. (2020). The discourse and coordination among advocacy coalitions: the case of Belo Monte. RAUSP Management Journal, 55(1), 86-99.

Weible, C. M. (2008). Expert-based information and policy subsystems: A review and synthesis. Policy Studies Journal, 36(4), 615-635.

Weible, C. M., \& Sabatier, P. A. (2005). Comparing policy networks: Marine protected areas in California. Policy Studies Journal, 33(2), 181-201.

Weible, C. M., Sabatier, P. A. \& McQueen, K. (2009). Themes and variations: Taking stock of the advocacy coalition framework. Policy Studies Journal, 37(1), 121-140.

Weiss, C. H. (1979). The Many Meanings of Research Utilization. Public Administration Review, 39(5), 426-431.

Weiss, C. H. (1986). The Circuitry of Enlightenment: Diffusion of Social Science Research to Policymakers. Science Communication, 8(2), 274-281.

Weiss, C. H. (1988). Evaluation for decisions: Is anybody there? Does anybody care? Evaluation Practice, 9(1), 5-19.

Weiss, C. H. (1998). Have We Learned Anything New About the Use of Evaluation? American Journal of Evaluation, 19(1), 21-33.

Wong, N. W. M. (2016). Environmental protests and NIMBY activism: Local politics and waste management in Beijing and Guangzhou. China Information, 30(2, SI), 143-164. 
RAP | Aprendizado e mudança em políticas públicas: explorando possibilidades no Modelo de Coalizões de Defesa

\section{Janaina Ma}

https://orcid.org/0000-0003-3646-3720

Aluna de doutorado no Programa de Pós-Graduação em Administração da Universidade de Brasília (PPGA / UnB); Pesquisadora do Instituto Nacional de Estudos e Pesquisas Educacionais Anísio Teixeira / Ministério da Educação (Inep/MEC) e do Grupo de Pesquisa "Governo e Políticas Públicas” do PPGA / UnB.

E-mail: janainafma@gmail.com

\section{Diego Mota Vieira}

http://orcid.org/0000-0003-1275-1648

Professor do Programa de Pós-Graduação em Administração e do Departamento de Administração da Universidade de Brasília; Líder do Grupo de Pesquisa “Governo e Políticas Públicas” do PPGA / UnB.

E-mail: diego1mv@yahoo.com.br 